\title{
Decreased electroencephalogram alpha band [8-13 Hz] power in amyotrophic lateral sclerosis patients: A study of alpha activity in an awake relaxed state
}

\author{
Jayashree Santhosh, Manvir Bhatia*, Shweta Sahu, Sneh Anand \\ Centre for Biomedical Engineering, Indian Institute of Technology, Delhi, Hauz Khas, New Delhi - 110 016, *Department of Neurology, All \\ India Institute of Medical Sciences, Ansari Nagar, New Delhi - 110 029, India
}

An attempt was made to quantitatively analyze the alpha activity in the awake relaxed state of Amyotrophic Lateral Sclerosis (ALS) patients and was compared with normals. ALS patients showed significantly low amplitude with a corresponding alpha band $(8-13 \mathrm{~Hz})$ power reduction, in both hemispheres though the change was more prominent in the left hemisphere. A review of the literature revealed no studies done on alpha oscillations in ALS patients; hence the results may have important implications for the interpretation of resting state brain activities.

Key Words: Amyotrophic Lateral Sclerosis (ALS), alpha rhythm amplitude, non-stimulus conditions, intra-cortical processes

\section{Introduction}

Various studies on Amyotrophic lateral sclerosis (ALS) patients using neuropathology, ${ }^{[1]}$ neuropsychology ${ }^{[2]}$ and neurophysiology ${ }^{[3-5]}$ have revealed that there is cortical dysfunction beyond the motor cortex. These studies were based on auditory selective attention, verbal fluency, word generation and/ or planning ability. No studies on alpha oscillations, which is reported to be a reflector of thalamic activity, ${ }^{[6]}$ have been performed. Hence in the present study, the alpha rhythms under non-stimulus condition, with eyes closed was studied and compared with controls.

\section{Case Report}

Twelve ALS patients (9 males, 3 females with mean age 46.75 years $(S D=14.429)$, mean disease duration - 14.33 months $(S D=$ 11.523)) diagnosed by a neurologist at a tertiary care center, and their age-matched controls ( 9 males, 3 females with mean age 47.17 years $(S D=15.320))$ were investigated, from March 2003 to March 2004. All patients with secondary cause were excluded and it was also made sure that no patient was on medication relevant to the central nervous system functions and none had dementia as per history. Ethical clearance was taken and all subjects gave their written consent to the experiment.

In the experimental paradigm designed for the study, the subject was asked to lie down comfortably in a relaxed position with eyes closed, and a total of 30-minute data was recorded from eight electrodes (C3, C4, P3, P4, F3, F4 and T3, T4). The recorded data from each subject was then divided into 5-second epochs, each extracting two from the start, two from the middle and two towards the end of the entire recording, for the purpose of analysis. Recording was done using Medelec Profile VEEG Machine (Oxford Instruments Medical Ltd.) as per the following settings: High Frequency filter $50 \mathrm{~Hz}$, Low Frequency filter $1.6 \mathrm{~Hz}$, Notch filter $50 \mathrm{~Hz}$, Sensitivity 7 micro Volt/mm and Sampling rate of $256 \mathrm{~Hz}$. From each of the 5-second epochs, for every one-second data, the FF'T spectrum of the 8-13 $\mathrm{Hz}$ alpha band was extracted for further analyses. All evaluations were done using MATLAB R13 v 6.5 signal processing toolbox and one way within subjects ANOVA (repeated measures) were done for statistical analyses using the Analyse-It software version 1.71 .

\section{Discussion}

Total band power values obtained for each unipolar electrode pair for central, frontal, temporal and parietal between ALS patients and their age-matched controls are shown in Figure 1 and 2 and Table 1.

Figures show that the maximum and minimum band power values for ALS patients lie between $190-7400 \mu \mathrm{V}^{2}$, whereas the same for Controls are between $280-23000 \mu \mathrm{V}^{2}$. From Table 1, the total alpha band power in the left hemisphere appeared to be about $60 \%$ - $70 \%$ lower in ALS as compared to that in age-matched controls. The $P$-values show that EEG alpha band power reduction was more significant in the left 


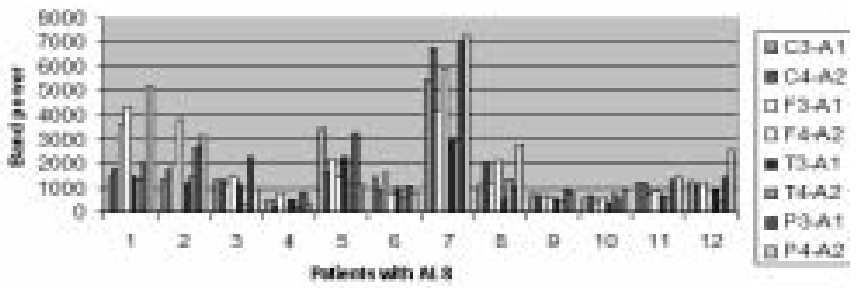

Figure 1: Total alpha band power values in microvolt ${ }^{2}$ from each electrode pair in 12 ALS patients calculated from a one-second EEG

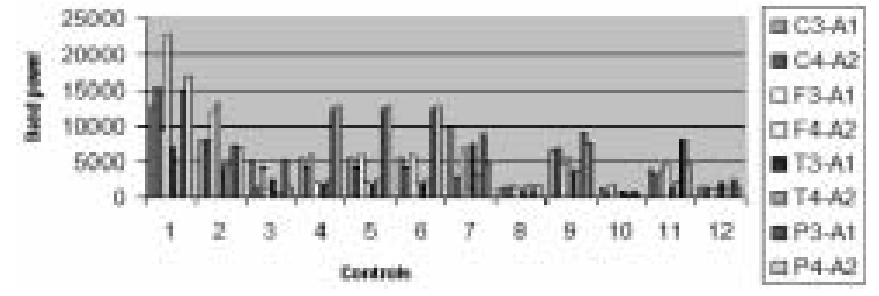

Figure 2: Total alpha band power values in microvolt ${ }^{2}$ from each electrode pair in 12 age-matched controls calculated from a onesecond EEG data

\begin{tabular}{|c|c|c|c|c|c|c|}
\hline \multirow{2}{*}{$\begin{array}{l}\text { Electrode } \\
\text { Site }\end{array}$} & \multirow{2}{*}{$\begin{array}{l}\text { ALS Patients } \\
\text { Mean (S.D.) }\end{array}$} & & \multicolumn{2}{|c|}{$95 \% \mathrm{Cl}$ of Mean } \\
\hline & & Mean (S.D.) & & & ALS & Controls \\
\hline C3-A1 & $1660.83(1406.98)$ & $5540.50(3531.94)$ & 1 & 0.0015 & 766.88-2554.79 & $3296.41-7784.59$ \\
\hline T3-A1 & 1160.33 (772.64) & $2978.17(2296.54)$ & 1 & 0.0067 & $669.42-1651.25$ & $1519.02-4437.32$ \\
\hline P3-A1 & $2050.33(1740.93)$ & 7853.92 (4728.62) & 1 & 0.0013 & $944.20-3156.47$ & $4849.50-10858.34$ \\
\hline $\mathrm{C} 4-\mathrm{A} 2$ & $1685.58(1693.91)$ & $4513.08(4088.24)$ & 1 & 0.0509 & 609.32-2761.85 & $1915.54-7110.62$ \\
\hline P4-A2 & 2285.50 (2101.14) & 7047.00 (5544.67) & 1 & 0.0171 & $950.50-3620.50$ & $3524.09-10569.91$ \\
\hline
\end{tabular}

Comparison of total alpha band power values in $\mu \mathrm{V}^{2}$ from each electrode pair calculated cumulatively for $12 \mathrm{ALS}$ and their age-matched controls, from a onesecond recorded data per individual; ${ }^{*} \mathrm{Cl}$ - Confidence Interval, $†$ DF- Degrees of Freedom

hemisphere ( $P$ values ranged from 0.0007 - 0067), compared with the right hemisphere $(P$ values ranged from 0.0030 $0.1208)$, though the changes appeared bilateral. It was also observed that the total alpha band power, compared between the left and right hemisphere within ALS patients, showed no significant changes.

Numerous studies have stated that the alpha activity is an indicator of memory and cognition, ${ }^{[7,8]}$ with thalamo-cortical and cortico-cortical networks playing an important role in the generation of alpha rhythmicity. It was reported that there are important cortical mechanisms responsible for spreading alpha activity over the cortex. ${ }^{[6]}$ Experiments done in patients with Alzheimer's, Dementia, and Aging ${ }^{[9-11]}$ had already reported significantly lower alpha power in resting EEG and observed alpha band abnormality in non-stimulus conditions. Since a review of the literature showed no such attempts made in ALS patients, the present experiment is a first step towards finding the alpha rhythm variations in resting conditions in ALS patients. The authors would like to mention that no occipital electrodes were used to quantify the occipital alpha rhythm, which is a limitation of the present study. The power reduction of alpha activity extracted from the central, frontal, parietal and temporal electrodes during non-stimulus conditions, when the subject is awake with eyes closed could be used as a marker for cortical involvement in extra motor manifestations of ALS patients. The findings in general agree with the earlier reported studies of reduction in alpha activity at rest in other neurological disorders, hence may have important implications for the interpretation of resting state cortical activity in ALS patients. The preliminary results show a possible disruption of intrinsic cortical neural connections in
ALS patients, and further studies are recommended in a much larger sample.

The authors would like to extend their approach to study the change or shift in total power in different spectral frequencies and to have more observations towards overall organization/disorganization or any loss of cortical rhythms in ALS patients.

\section{Acknowledgement}

The authors acknowledge their gratitude to Department of Science and Technology (DST), Government of India for providing financial help for the study. The authors also thank the scientific and technical staff at rehabilitation laboratory, Indian Institute of Technology, Delhi and Clinical Neurophysiology laboratory, All India Institute of Medical Sciences, New Delhi for the help in carrying out the experiment.

\section{References}

1. Nagy D, Kato T, Kushner PD. Reactive astrocytes are wide spread in the cortical gray matter of amyotrophic lateral sclerosis. J Neurosci Res 1994;38:33647.

2. Hanagasi HA, Gurvit IH, Ermutlu N, Kaptanoglu G, Karamursel Sacit, Idrisoglu HA, et al. Cognitive impairment in amyotrophic lateral sclerosis: Evidence from neuropsychological investigation and event related potentials. Brain Res Cogn Brain Res 2002;14:234-44.

3. Vieregge P, Wauschkuhn B, Heberlein I, Hagenah J, Verleger R. Selective attention is impaired in amyotrophic lateral sclerosis - a study of event-related EEG potentials. Brain Res Cogn Brain Res 1999;8:27-35.

4. Santhosh J, Bhatia M, Sahu S, Anand S. Quantitative EEG analysis for assessment to 'plan' a task in amyotrophic lateral sclerosis patients: A study of executive functions (planning) in ALS patients. Brain Res Cogn Brain Res 2004;22:59-66.

5. Abrahams S, Leigh PN, Harvey A, Vythelingum GN, Grise D, Goldstein LH Verbal Fluency and executive dysfunction in amyotrophic lateral sclerosis (ALS). Neuropsychologia 2000;38:734-47.

6. Lopes da Silva FH, Vos JE, Mooibroek J, Van Rotterdam A. Relative contributions of intracortical and thalamo-cortical processes in the generation of alpha rhythms, revealed by partial coherence analysis. Electroencephalogr Clin Neurophysiol 1980;50:449-56.

7. Klimesch W. EEG alpha and theta oscillations reflect cognitive and memory 
performance: A review and analysis. Brain Res Brain Res Rev 1999;29:169-95.

8. Brunia CHM. Thalamo-cortical relations in attention and consciousness. Int .J Psychophysiol 2001;43:1-4.

9. Wada Y, Nanbu Y, Jiang ZY, Koshino Y, Yamaguchi N, Hashimoto T Electroencephalographic Abnormalities in Patients with Presenile Dementia of the Alzheimer Type: Quantitative Analysis at Rest and during Photic Stimula- tion. Biological Psychiatry 1997;41:217-25.

10. Knott V, Mohr E, Mahoney C, Ilivitsky V. Quantitative electroencephalography in Alzheimer's disease: comparison with a control group, population norms and mental status. J Psychiatry Neurosei 2001;26:106-16.

11. Yener GG, Leuchter AF, Jenden D, Read SL, Cummings JL, Miller BL. Quantitative EEG in fronto-temporal dementia. Clin Electroencephalogr 1996;27:61-8.

Accepted on 24.12.2004 